\title{
Oral-Facial-Digital Syndrome with Hirschsprung Disease - A New horizon
}

\author{
Roopak Dubey ${ }^{1}$, Kamal Kumar Sen ${ }^{2}$, Rudra Narayan Dash ${ }^{3}$, Mayank Goyal ${ }^{4}$ \\ ${ }^{1} 1$ st Year Post Graduate Trainee, ${ }^{2}$ Professor and HOD, ${ }^{3}$ Associate Professor, ${ }^{4} 1$ st Year Post Graduate Trainee, Department of \\ Radio-diagnosis, Kalinga Institute of Medical Sciences, Bhubaneswar, Odisha, India
}

Corresponding author: Dr. Roopak dubey, Department of Radio-diagnosis, Kalinga Institute of Medical Sciences, KIIT Road, Patia, 751024, Bhubaneswar, India

OdishaDOI: http://dx.doi.org/10.21276/ijcmsr.2020.5.1.34

How to cite this article: Roopak Dubey, Kamal Kumar Sen, Rudra Narayan Dash, Mayank Goyal. Oral-facialdigital syndrome with hirschsprung disease - a new horizon. International Journal of Contemporary Medicine Surgery and Radiology. 2020;5(1):A155-A157.

\section{A B S T R A C T}

Introduction: Oral-facial-digital syndrome(OFDS) is a rare disorder and its association with Hirschsprung disease (HSCR) makes it further more infrequent. The main aim and objective of this paper is to enhance the understanding between Hirschsprung disease and Oral facial digital syndrome.

Case report: We are presenting here a 8 months old baby boy with biopsy proven Hirschsprung disease along with morphological anomalies consistent with Oral-Facial-Digital syndrome. A previously undescribed case of Hirschsprung disease with post axial polydactyly, hypertelorism, ASD, hyperplastic frenulum, high arched palate, depressed nasal bridge, low set ears and frontal bossing is presented here.

Conclusion: The presented case showed some similarities to "Unclassified variant" of OFDS but there were some differences also which had not been described in the literature earlier. Hence, this case can be considered either as an extended version of "Unclassified variant" of OFDS or a new variant of OFDS.

Keywords: Oral-facial-digital Syndrome, Hirschsprung Disease, Variant

\section{INTRODUCTION}

Oral-facial-digital syndrome (OFDs) is a group of rare disorders characterised by abnormalities of oral cavity, face and digits of hands and feet ${ }^{1}$.Its association with Hirschsprung disease makes it further uncommon.

The relationship of ciliopathies like Oral-facial-digital syndrome, Joubert syndrome and Meckel Gruber Syndrome with neurocristopathy like Hirschsprung disease (HSCR) has been described in the literature, however there is lack of clear understanding between their association and finding of a common genetic/molecular associating link between the two is under progress.

A rare association of Hirschsprung disease with probably an extended version of unclassified variant of OFDs or a new variant of OFDs is presented in this report.

\section{CASE REPORT}

A 8 months old very irritable male baby from nonconsanguineous parents, was brought to our Institution with bilious vomiting and abdominal distention for 12 hours. His mother stated that the infant had been constipated since birth and failed to pass meconium during the first 48 hours of life. Distended bowel loops were noted on X-ray (Fig.1). A subsequent rectal biopsy revealed absence of ganglionic cells in colonic wall, confirming the diagnosis of Hirschsprung disease. He was treated surgically by "Duhamel's retro- rectal pull through" procedure. There was no family history of congenital anomalies.

Besides Hirschsprung disease, several morphological congenital defects were also noted. Left hand showed postaxial polydactyly where two digits arising from $4^{\text {th }}$ metacarpal (Fig-2A). In addition there was $Y$ shaped $3^{\text {rd }}$ and small underdeveloped $4^{\text {th }}$ metacarpal with post-axial polydactyly in right hand (Fig.2B). Left foot showed $\mathrm{Y}$ shaped $4^{\text {th }}$ metatarsal and post-axial polydactyly (Fig.2C). So, digital features include post-axial polydactyly in all the limbs except right foot.

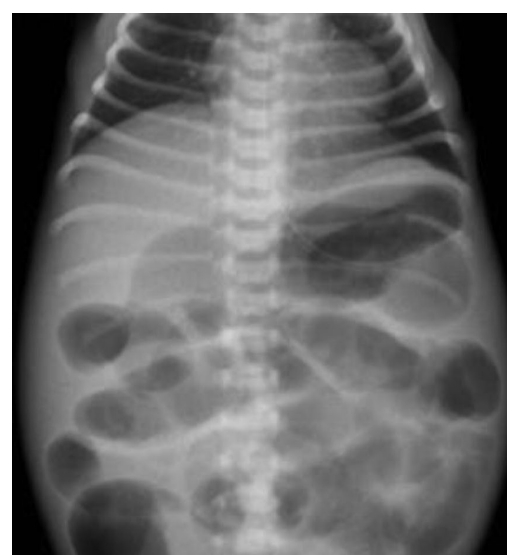

Figure-1: Pre-operative radiograph of abdomen showing distended bowel loops. 


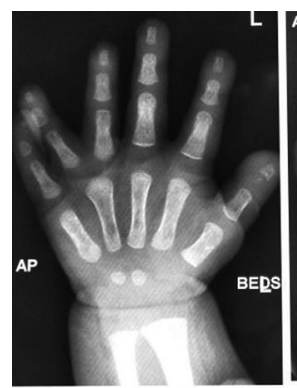

$2 \mathrm{~A}$
2B

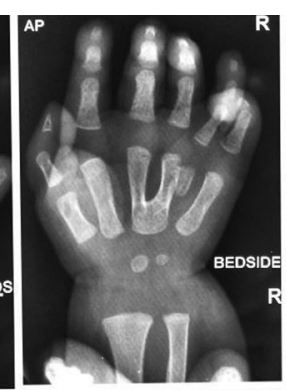

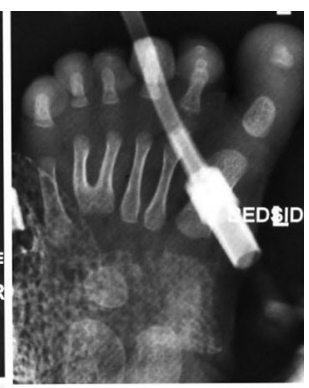

2C
Figure-2: (A). Radiograph of left hand -AP view showing post axial polydactyly of left hand. Note that two digits arising from 4th left metacarpaL. (B). Radiograph of right hand-AP view showing post-axial polydactyly of right hand with Y-shaped 3rd right metacarpal. Also note the right 4th under-developed metacarpal. (C). Radiograph of left footAP view showing post axial polydactyly. Also note Y-shaped 4th metatarsal.

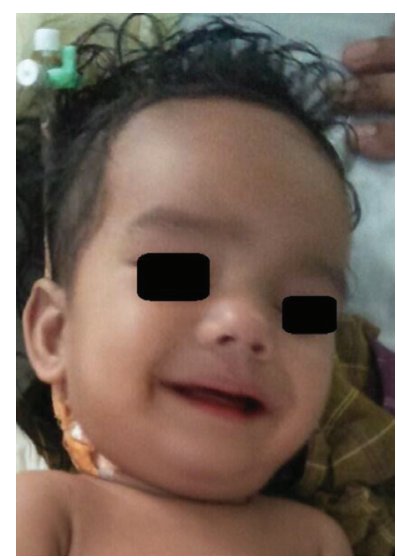

Figure-3: Showing Wide and depressed nasal bridge, hypertelorism, frontal bossing,low set ears and thick hair.

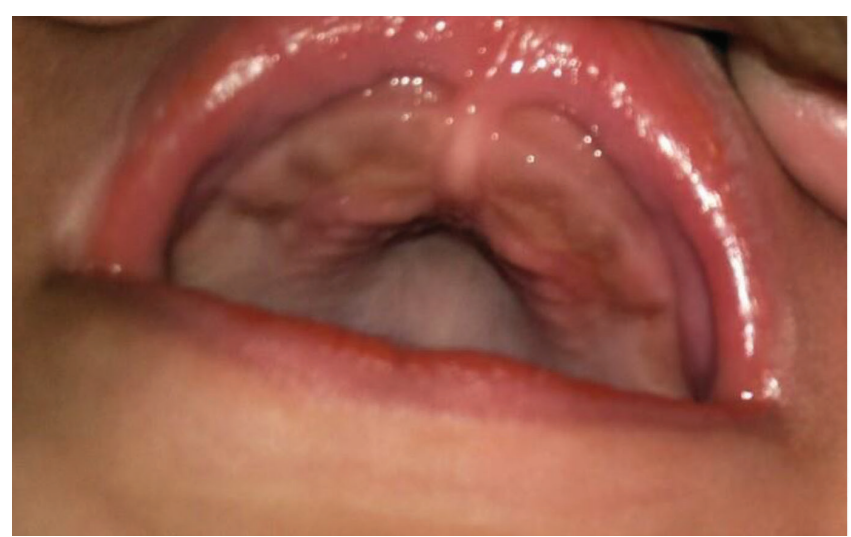

Figure-4: Showing Hyperplastic frenulum and High arched palate.

Oro-facial features included frontal bossing, depressed nasal bridge, hypertelorism, low-set ears (Fig-3). Thick hair, hypertrophic gums, high arched palate \& hyperplastic frenulum (Fig-4) were also noted. ECHO cardiography was consistent with the presence of Atrial Septal Defect. Mild intellectual disability and developmental milestone delay was also seen. Ultrasonography (USG) of abdomen did not reveal any abnormality. Chromosomal studies showed that he had a

\begin{tabular}{|l|c|c|}
\hline Anomalies & $\begin{array}{c}\text { Unclassified } \\
\text { variant of OFDS }\end{array}$ & $\begin{array}{c}\text { Presented } \\
\text { case }\end{array}$ \\
\hline Fused kidneys & + & - \\
\hline TOF/VSD/ASD & TOF/VSD & ASD \\
\hline Corpus callosum agenesis & + & - \\
\hline Intellectual disabilities & ++ & + \\
\hline Lobulated tongue & + & - \\
\hline Hyperplastic frenulum & - & + \\
\hline Cleft palate & + & - \\
\hline High arched palate & - & + \\
\hline Low set ears & - & + \\
\hline \multicolumn{2}{|l|}{ Table-1: Variations in association of congenital anomalies with } \\
\multicolumn{2}{|l}{ HSCR } \\
\hline
\end{tabular}

normal male chromosomal pattern of $46 \mathrm{XY}$.

\section{DISCUSSION}

Hirschsprung disease presents with associated chromosomal anomalies in $12 \%$ cases and additional congenital anomalies in $18 \%$ cases $^{2}$. Common associated congenital anomalies with Hirschsprung disease include cleft palate, polydactyly, cardiac septal defects, Gastro-intestinal malformations and craniofacial anomalies ${ }^{3}$. The variants that have been recorded so far are:

1. HSCR with polydactyly, unilateral renal agenesis, hypertelorism and congenital deafness. ${ }^{4}$

2. HSCR with post-axial polydactyly and ventricular septal defect. $^{5}$

3. HSCR with hypoplasia of distal phalanges and nails and mild dysmorphic features. ${ }^{6}$

4. HSCR with preaxial polydactyly, heart defects and laryngeal anomalies. ${ }^{7}$

5. HSCR with brachydactyly, macrocephaly and vertebral anomalies. $^{8}$

6. HSCR with brachydactyly type D. ${ }^{9}$

To the best of our knowledge, the present case has a galaxy of associated congenital malformations which has not been described in literature earlier. The closest diagnosis favouring "Unclassified variant of OFDs" presents with post-axial polydactyly, fused kidneys, TOF/VSD, lobulated tongue, cleft palate, corpus callosum agenesis, moderate intellectual disabilities and Hirschsprung disease. In contrast to unclassified OFDs which presents with fused kidneys, our patient showed normal USG study without any renal anomalies. ASD was found in our patient in contrast to TOF/ VSD, a component of unclassified OFDs. Furthermore, instead of lobulated tongue and cleft palate usually present in unclassified variant, our patient had hyperplastic frenulum and high arched palate.The differentiating features between this case and Unclassified variant of OFDs are given in the table-1.

\section{CONCLUSION}

Compiling the features in our patient, it was obvious that it doesn't fit into any of the category of OFDs. The closest subtype is the Unclassified variant of OFDs. Hence we believe that this could be an extended version of Unclassified variant or a previously undescribed variant of OFDs. Absence of 
positive family history in this case implies that the OFD malformations could have resulted from a spontaneous mutation, an X-linked dominant trait or an autosomal recessive trait with minimal expression in the preceding generations.

\section{REFERENCES}

1. Silva Sousa YTC, Kanaan DDM. The Oro-facialdigital syndrome- Manifestations in the oral cavity -Case report. Braz Dent J. 1995;5(1): 71-74.

2. J Amiel, E Sproat-Emison, M Garcia-Barcelo. Hirschsprung disease, associated syndromes and genetics: a review.J Med Genet 2008;45(3):1-14.

3. Spouge D, Baird PA. Hirschsprung disease in a large birth cohort. Teratology 1985;32(2):171-7.

4. Brooks AS, Breuning MH, Meijers C. Spectrum of phenotypes associated with Hirschsprung disease: an evaluation of 239 patients from a single institution. The Third International Meeting: Hirschsprung disease and related neurocristopathies.Evian, France, 1998.

5. Laurence KM, Prosser R, Rocker I, Pearson JF, Richard C. Hirschsprung's disease associated with congenital heart malformation, broad big toes, and ulnar polydactyly in sibs: a case for fetoscopy. J Med Genet 1975;12(4):334-8.

6. al-Gazali LI, Donnai D, Mueller RF. Hirschsprung's disease, hypoplastic nails, and minor dysmorphic features: a distinct autosomal recessive syndrome? J Med Genet 1988;25(1):758-61.

7. Huang T, Elias ER, Mulliken JB, Kirse DJ, Holmes LB. A new syndrome: Heart defect, laryngeal anomalies, preaxial polydactyly, and colonic aganglionosis in sibs. Genet Med 1999;1(5):104.

8. Toriello HV, Komar K, Lawrence C, Higgins JV, Waterman DF. Macrocephaly, Hirschsprung disease, brachydactyly, vertebral defects, and other minor anomalies. Dysmorphology and Clinical Genetics 1988;1(4):155-7.

9. Reynolds JF, Barber JC, Alford BA, Chandler JG, Kelly TE. Familial Hirschsprung's disease and type D brachydactyly: a report of four affected males in two generations. Pediatrics 1983;71(6):246-9.

Source of Support: Nil; Conflict of Interest: None

Submitted: 29-11-2019; Accepted: 20-12-2019; Published online: 28-02-2020 